LA-UR-01-2637

Approved for public release; distribution is unlimited.

\section{Title: STRUCTURE AND ELECTRONIC PROPERTIES OF SELF-ASSEMBLED MACROCYCLE AND POLYMER MULTILAYERS}

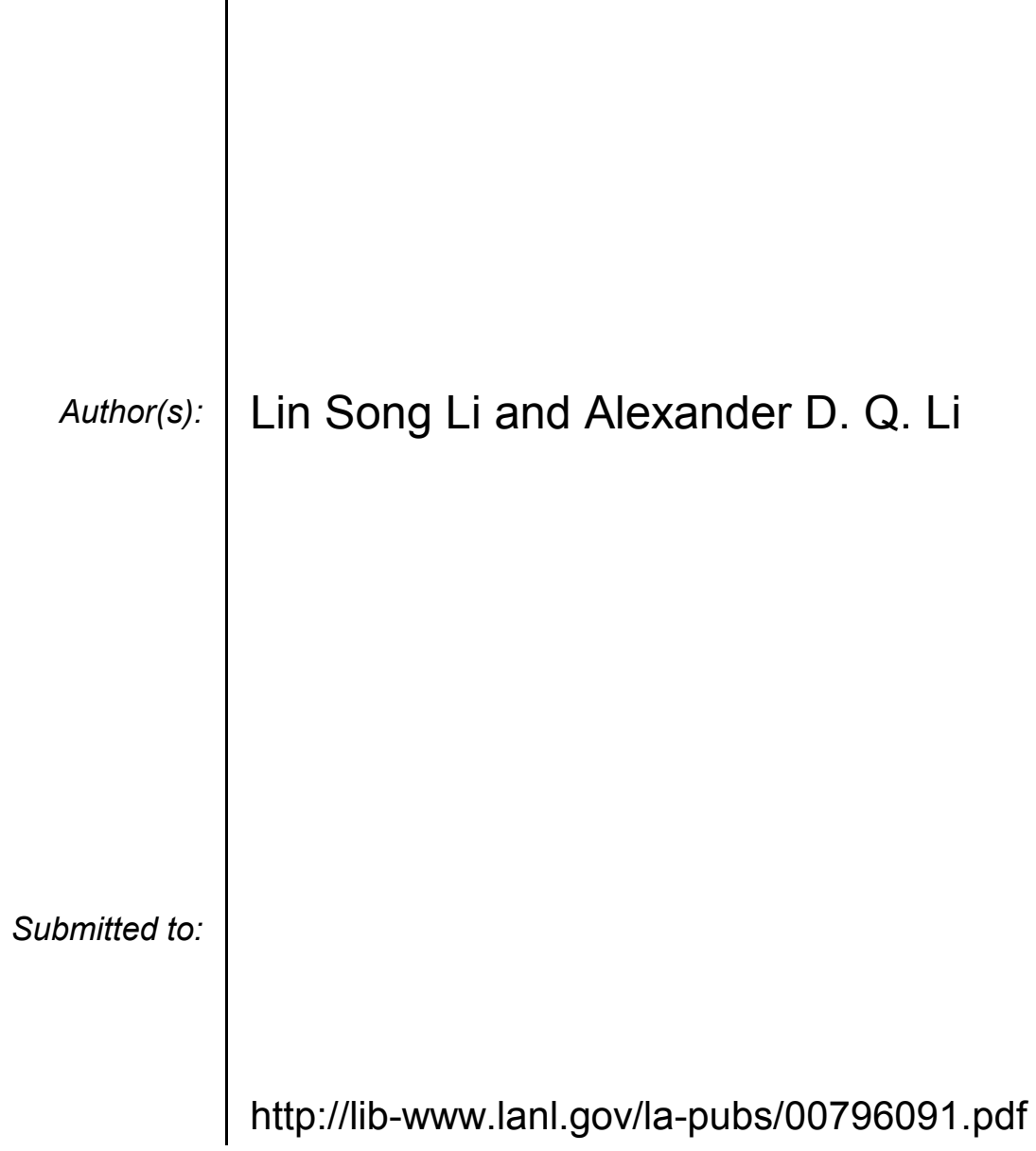




\title{
STRUCTURE AND ELECTRONIC PROPERTIES OF SELF-ASSEMBLED MACROCYCLE AND POLYMER MULTILAYERS
}

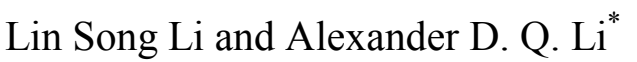

\section{INTRODUCTION}

Layer-by-layer (LBL) self-assembly technique has been widely used to fabricate artificial thin films for a number of potential applications with several advantages (Decher, 1997; Lenahan et al., 1998; Kaschak et al., 1999). First, many materials with charges can be chosen as building blocks for this simple approach to thin-film fabrication; this includes conducting polymers, optical chromophores, metal complexes, or semiconductor nanoparticles. Second, LBL self-assembly processes are much simpler when compared to the Langmuir-Blodgett technique, or chemical/physical vapor depositions (Liu et al., 1997; Li et al., 1998; Lütt, et al., 1998; Dante et al., 1999; Kim et al., 1999; Liu et al., 1999; Ostrander et al., 2001). Finally, by controlling length scale at the nanometer level, LBL approach can be used to construct materials with designed properties for applications in electronics, photonics, and optoelectronics. Since the interfaces between each layer become the key linkages for multilayers and therefore interface properties are crucial to the self-assembled systems (Li et al., 1998; Schlenoff et al., 1998).

The Kelvin Probe, as a non-contact, non-destructive vibrating capacitor device, has been used to measure the work function difference, or for non-metals, the surface potential, between a conducting specimen and a vibrating tip (Lüth, 1993; Baikie and Estrup, 1998). Measuring surface potentials of organic monolayers on conducting or seminconducting solids has been carried out successfully (Bruening et al., 1994; Bruening et al., 1995; Bruening et al., 1997; Bastide et al., 1997; Cohen et al., 1997;

* Lin Song Li, MST-STC, Los Alamos National Laboratory, Los Alamos, NM 87545, Tel: 505-665-9089; Fax: 505-667-8021; E-mail: linsong@lanl.gov. Alexander DeQuan Li, Department of Chemistry and Center for Materials Research, Washington State University, Pullman, WA 99164, Tel: 509-335-7196; E-mail: dequan@wsu.edu. 
Cohen et al., 1999; Kronik and Shapria, 1999; Cohen et al., 2000). Studies on monitoring work function change in situ were also reported (Hamma and Cabarrocas, 1999; Tadayyon et al., 1999). Cahen D. and co-workers reported surface work function control of a semiconductor through molecular modification of its surfaces (Bruening et al., 1995; Bruening et al., 1997; Bastide et al., 1997; Cohen et al., 1997; Cohen et al., 1999; Cohen et al., 2000). Their results indicated that surface electric properties were a function of molecular modifiers in systems composed of chemisorbed monolayers of dicarboxylic acids on $\mathrm{CdTe}, \mathrm{CdSe}, \mathrm{CuInSe} \mathrm{H}_{2}, \mathrm{GaAs}$ and InP. Recently, we employed Kelvin probe technique to measure work functions of organic and polymeric multilayers grown on conductive substrates ( $\mathrm{Li}$ et al., 2000). The results indicated that the variation of the work function, as multilayers of PDDA [poly(dimethyldiallyammonium chloride)] and NiPc are self-assembled, is periodic.

In this chapter, we choose to assemble multilayer thin films using positively charged oligomeric viologen and negatively charged nickel phthalocyanine (NiPc) on a conductive oxide (Figure 1). Due to their interesting chemical structure and electronic properties, phthalcyanines have received considerable attention as constituents in optoelectronic devices (Hanack and Lang, 1994; Smolenyak et al., 1999), such as a single molecular dye Schottky barrier or hole conductors (p-type layers) in heterojunction devices. Conversely, viologens are electron acceptors (Kelly and Rodgers, 1994; Gong et al., 2000; Le et al., 2000), which seem ideal when coupled with electron-rich phthalocyanines. We hope that the study of the effect of self-assembled multilayers on the work function will provide insightful information on materials design and lead to the control of electronic properties on surfaces through molecular engineering.

\section{EXPERIMENTAL SECTION}

\subsection{Materials Synthesis}

Oligo(hexylene-4,4'-bipyridinum dibromide), or OHBP, was synthesized in our laboratory according to the following procedures. Bipyridine $(0.8698 \mathrm{~g})$ was mixed with 1,6-dibromohexane $(1.3587 \mathrm{~g})$ in approximately $2 \mathrm{~mL}$ of ethanol. The clear transparent mixture was then heated to $100{ }^{\circ} \mathrm{C}$ for about 24 hours. After reaction, a yellow solid was collected and washed with more ethanol, yielding $2.223 \mathrm{~g}$ (yield: $100 \%$ ) of productoligo(hexylene 4,4'-bipyridinum dibromide), or oligomeric viologen. ${ }^{1} \mathrm{H}$ NMR (DMSO$\left.\mathrm{d}_{6}\right), \delta 1.35(\mathrm{~m}, 4 \mathrm{H}), 2.03(\mathrm{~m}, 4 \mathrm{H}), 4.71(\mathrm{~m}, 4 \mathrm{H}), 8.78(\mathrm{~d}, 4 \mathrm{H}), 9.53(\mathrm{~d}, 4 \mathrm{H})$. The average degree of polymerization is 11 . NiPc was purchased from Aldrich and used without further purification.
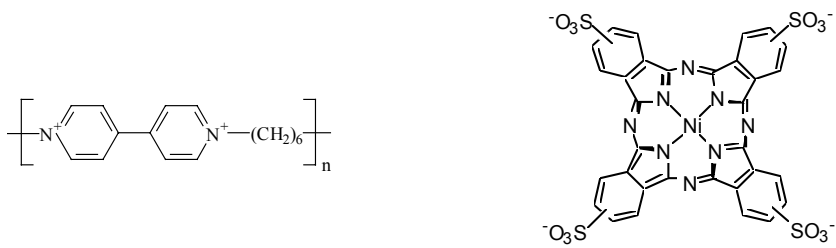

Figure 1. Molecular structures of oligo(hexylene 4,4'-bipyridinum dibromide), or OHBP (left) and nickel phthalocyaninetetrasulfonic acid tetrasodium salt, or NiPc (right). 


\subsection{Preparation of Self-Assembled Multilayers}

Positively charged OHBP and negatively charged NiPc were used for preparing selfassembled bilayers. The concentrations of OHBP polymer and NiPc aqueous solution were $50 \mathrm{mM}$ and $1 \mathrm{mM}$, respectively. The ITO substrates (resistivity $\leq 40 \Omega / \mathrm{cm}^{2}$ ) were purchased from Delta Technologies Company. Before using them, they were cleaned for $10 \mathrm{~min}$ by ultrasonication in an acetone solution. Then, the substrates were rinsed several times in deionized water and dried by $\mathrm{N}_{2}$. Immediately after drying, the substrates were immersed in the OHBP solution for about $5 \mathrm{~min}$. The attractive force between positively charged OHBP polymer and negatively charged (OH-terminated) ITO surface is expected to coat the ITO surface with an OHBP monolayer. The coated sample then was washed with deionized water to remove all of the non-adsorbed materials and dried under $\mathrm{N}_{2}$ for measurements. Then, the OHBP-coated substrate was immersed in a NiPc aqueous solution for another $2 \mathrm{~min}$, followed by washing with deionized water. At this time, an OHBP-NiPc bilayer was formed on the ITO substrate. It was dried under $\mathrm{N}_{2}$ again for further measurements. Repeating the above steps, additional bilayers of OHBP and NiPc were deposited and a multilayer began to emerge on the ITO substrate.

\subsection{Instrumentation and Measurements}

All infrared spectra of OHBP-NiPC multilayers were collected on a Bio-Rad FT-IR spectrometer (Mode FTS-40) equipped with a MCT detector at $2 \mathrm{~cm}^{-1}$ resolution. The measurements were carried out at an external reflection configuration, polarized within the incident plane at a grazing angle of 70 degree. UV-visible spectra were collected with a Varian Cary 1C instrument.

A commercial Kelvin probe system (KP-6500 Digital Kelvin Probe System, McAllister Technical Services) was used to measure work function changes. This apparatus measures the contact potential difference (CPD) between a reference plate and the sample's surface. CPD is defined as the work function of the reference plate connected to the preamplifier minus the work function of the sample, i.e. $\phi_{\mathrm{m}}-\phi_{\mathrm{sam}}$ (where $\phi_{\mathrm{m}}$ is the work function of the reference plate (a constant), and $\phi_{\mathrm{sam}}$ is the work function of sample). By comparing the work function before and after deposition of OBHP or NiPc onto an ITO substrate, the change in work function, $\Delta \phi$, after the deposition is obtained. The probe plate is made of stainless steel with a diameter of $10 \mathrm{~mm}$. It was electrically connected via ground to the sample during the measurement with an accuracy of $2.5 \mathrm{mV}$. The typical distance between the reference plate of the probe and the sample was less than $\sim 1 \mathrm{~mm}$ at $100 \mathrm{~Hz}$ oscillation frequency (Li et al., 2000). A CPD measurement was obtained by the Kelvin Probe system under ambient conditions each time after the new monolayer (OBHP or NiPc) was deposited onto the ITO substrate.

Surface photovoltage spectroscopy (SPS) measurements were carried out on a homebuilt setup (Scheme 1). ITO was chosen as the substrates that were illuminated through the transparent backside by monochromatic light, which was obtained by passing light from a $450 \mathrm{~W}$ xenon lamp through a double-prism monochromator (SPEX FL3-21). The Kelvin probe was employed to pick up the photovoltage signal. Photo-induced surface photovoltage spectra were obtained by scanning the wavelength of the incident light through the UV and visible range $(250-710 \mathrm{~nm})$ at a typical scanning rate of $20 \mathrm{~nm} / \mathrm{min}$. The raw SPS data were not corrected for the spectrum of the xenon lamp. 


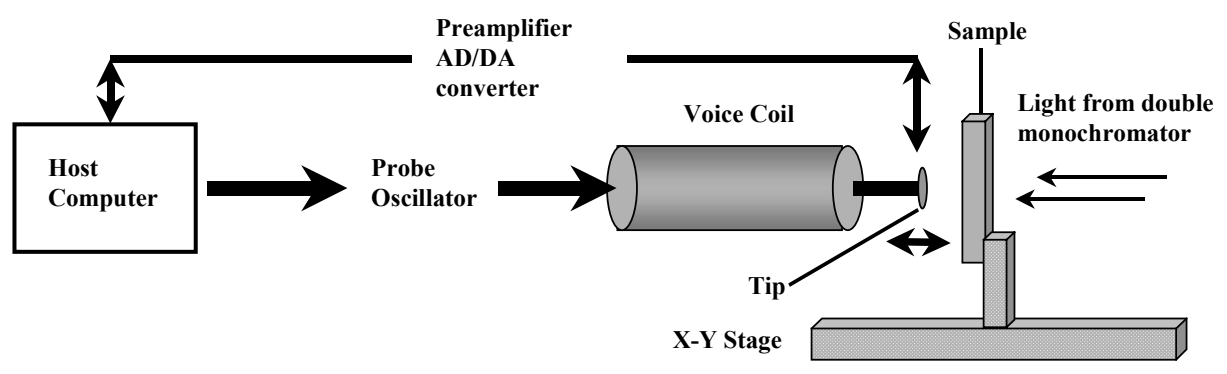

Scheme 1. Setup diagram for the measurements of surface photovoltage spectroscopy using Kelvin probe.

\section{RESULTS AND DISCUSSION}

ITO surfaces always show negatively charged characteristics due to the formation of dangling Sn- $\mathrm{O}^{-}$bonds. Other likely contributions of the negative charges can be attributed to the formation of a typical $n$-type depletion layer on bare ITO. Therefore, in an aqueous solution, positively charged OHBP forms a monolayer on the substrate surfaces through self-organization or association of charge pairs, a process that assembles solutionsolvated oligomers into monolayer films. Similarly, negatively charged NiPc macrocycles will bind to the dispersed positively charged oligomeric viologen monolayer. Multilayer thin films can be obtained by alternately dipping the substrate into the solutions of OHBP and NiPc. In the following discussions, we first establish the formation and structure of the OHBP and NiPc multilayers through optical absorption and surface infrared studies. Subsequently, we will monitor the evolution of the surface physical properties as each organic layer ( $\mathrm{OHBP}$ or $\mathrm{NiPc})$ is assembled.

\subsection{UV-vis Measurements on Multilayer Thin Films}

UV-vis spectra of OHBP-NiPc multilayers are shown in Figure 2. All the spectra exhibit the characteristic $\alpha$ and $\beta$ absorption at 634 and $670 \mathrm{~nm}$ due to the $\pi-\pi^{*}$ electronic transitions in NiPc molecules. The absorption at 274 and $343 \mathrm{~nm}$ are due to $\pi-\pi^{*}$ electronic transitions in viologens. The growth of the film was monitored by measuring the absorption of both OHBP at $274 \mathrm{~nm}$ and NiPc at $634 \mathrm{~nm}$. An approximate linear relationship between the optical absorption and the number of depositions was observed. This suggests that about the same amount of OHBP and NiPc macrocycle were deposited with each dipping if orientation effect of the chromophores is ignored. This assumption is generally acceptable because charge-charge interactions have little preference to molecular orientation. 


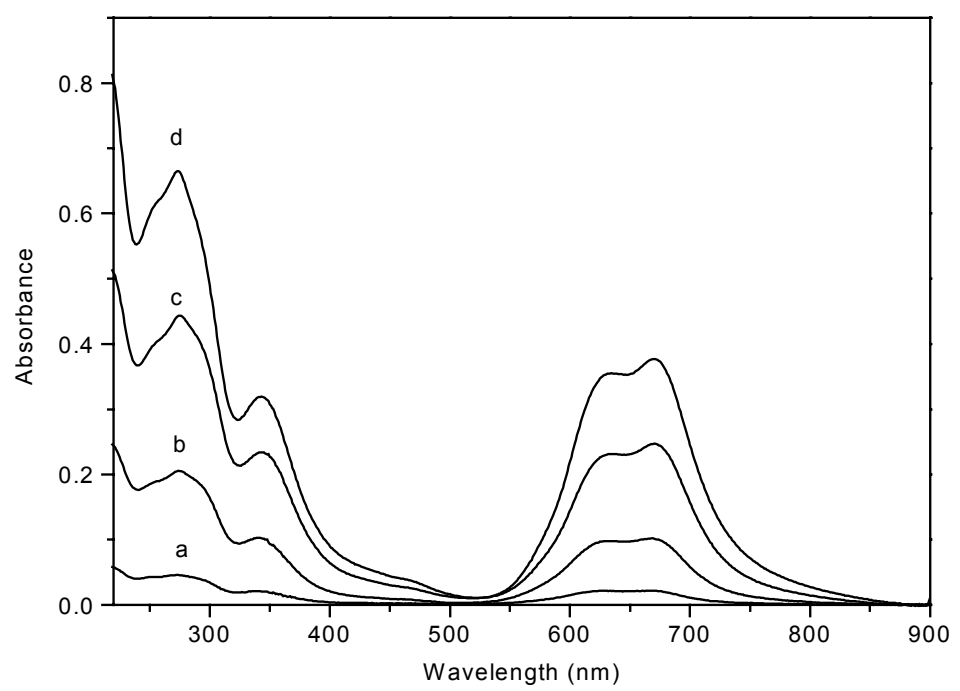

Figure 2. Optical absorption of (a) 3 layer-pairs of OHBP-NiPc; (b) 9 layer-pairs of OHBP-NiPc; (c) 15 layerpairs of OHBP-NiPc; (d) 21 layer-pairs of OHBP-NiPc on quartz substrates.

\subsection{Grazing Angle FTIR Studies on Self-assembled Multilayers}

To understand the self-assembled multilayer better, we have carried out FTIR measurements on both self-assembled films and bulk materials using grazing angle (70 degrees) external reflection of the IR beam from conductive ITO surfaces in a p-polarized geometry. In this configuration, only those IR vibration modes that have a non-zero component along the surface normal will be measured. FTIR spectroscopy of NiPc dropcasted film on ITO was reported by us previously ( $\mathrm{Li}$ et al., 2000). There are four bands at $1240\left(\mathrm{~s}, v_{\mathrm{as}}\right), 1202\left(\mathrm{~s}, \mathrm{v}_{\mathrm{as}}\right), 1106\left(\mathrm{w}, \mathrm{v}_{\mathrm{s}}\right)$, and $1030\left(\mathrm{w}, \mathrm{v}_{\mathrm{s}}\right) \mathrm{cm}^{-1}$ attributed to the sulfonate groups on NiPc (Colthup et al., 1975; Stymne et al., 1979; Watanabe et al., 1983; Enokida and Hirohashi, 1991; Fukui et al., 1991;). The macrocycle in-plane vibrations of C-N or C-C stretching modes bands are located at 1602, 1520, 1489, 1327, and $1089 \mathrm{~cm}^{-1}$, and the $1160 \mathrm{~cm}^{-1}$ band is due to the $\mathrm{C}-\mathrm{H}$ in-plane bending mode. On surfaces, the in-plane vibration modes of the NiPc are suppressed while the sulfonate bands remain strong $\left(\sim 1200 \mathrm{~cm}^{-1}\right)$. These results indicate that NiPc disk-like molecules are mostly lying flat on the surfaces in the drop-casted thin films.

Band assignment of OHBP is based on the model compound methyl viologen or bipyridinium (Christensen and Hamentt, 1989; Bae et al., 1991). The bands at 3122 and $3044 \mathrm{~cm}^{-1}$ are due to the aromatic Ar-H. Aliphatic C-H vibrations located at 2935 and $2863 \mathrm{~cm}^{-1}$, are assigned as asymmetric and symmetric methylene stretching modes, respectively. The methylene bending vibrations are located at 1468 and $1450 \mathrm{~cm}^{-1}$. The bending vibrations of $\mathrm{C}-\mathrm{H}$ from aromatic rings are located at 1348, 1233, and $1177 \mathrm{~cm}^{-1}$. The vibrations of $\mathrm{C}=\mathrm{N}$ or $\mathrm{C}=\mathrm{C}$ stretching modes including ring modes are located at 
$1639,1556,1508$, and $1252 \mathrm{~cm}^{-1}$, and the $834 \mathrm{~cm}^{-1}$ band is due to the N-alkyl stretching mode. The $1639 \mathrm{~cm}^{-1}$ band is characteristic of the pyridinium ring breathing mode, confirming the formation of oligomeric viologen.

For self-assembled multilayers, the surface IR spectra of $\mathrm{N}=9,18$ where $\mathrm{N}$ is the number of deposited OHBP-NiPc bilayers, are shown in Figure 4. With $\mathrm{N}=9$ OHBPNiPc bilayers, OHBP bands at 2925, 2858, 1638, and $811 \mathrm{~cm}^{-1}$, and NiPc bands at 1198 and $1033 \mathrm{~cm}^{-1}$ become visible. For the $\mathrm{N}=18$ sample, the bands belonging to OHBP and $\mathrm{NiPc}$ in the range of 1750 to $700 \mathrm{~cm}^{-1}$ are more distinct. Comparing with the bulk vibration information, a new band at $1227 \mathrm{~cm}^{-1}$ belonging to NiPc emerged; this band is originally from sulfonate groups and responsible for the formation of salt bridges of $\mathrm{SO}_{3}^{-} \cdots \mathrm{Py}^{+}-\mathrm{R}-$, which indicated the binding between OHBP and NiPc. In OHBP, both aromatic Ar-H and aliphatic $\mathrm{C}-\mathrm{H}$ vibrations experienced considerable shift. For example, a blue shift of $14 \mathrm{~cm}^{-1}$ from $3059 \mathrm{~cm}^{-1}$ to $3044 \mathrm{~cm}^{-1}$ occurred in the Ar-H mode. Both aliphatic C-H shifted from 2925 and $2858 \mathrm{~cm}^{-1}$ to 2935 and $2863 \mathrm{~cm}^{-1}$, corresponding to red shifts of $10 \mathrm{~cm}^{-1}$ and $5 \mathrm{~cm}^{-1}$, respectively. We believe that these shifts are also due to the formation of charge pairs $\left(-\mathrm{SO}_{3}{ }^{-} \cdots \mathrm{Py}^{+}-\mathrm{R}-\right)$ between viologens and $\mathrm{NiPc}$ sulfonates. The intensity of the OHBP bands at 2925 and $1638 \mathrm{~cm}^{-1}$ and that of the NiPc bands at 1326 and $1033 \mathrm{~cm}^{-1}$ were monitored during multilayer deposition. These four bands increased linearly with deposition number $\mathrm{N}$, suggesting that materials deposited during each cycle was approximately constant. This observation is consistent with those of optical absorption studies.

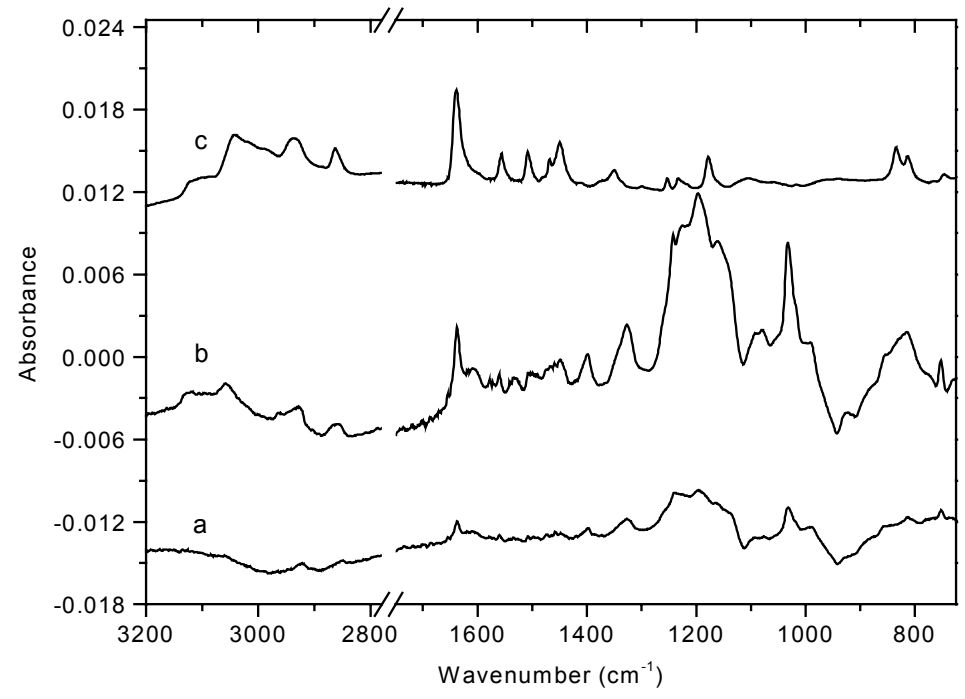

Figure 3. External gazing angle reflection (p-polarized; $70^{\circ}$ ) of infrared absorption spectra of self-assembled OHBP-NiPc multilayers on ITO substrates. (a) $N=9$ bilayer; (b) 18 bilayer; (c) OHBP drop casted films onto an ITO substrate. 


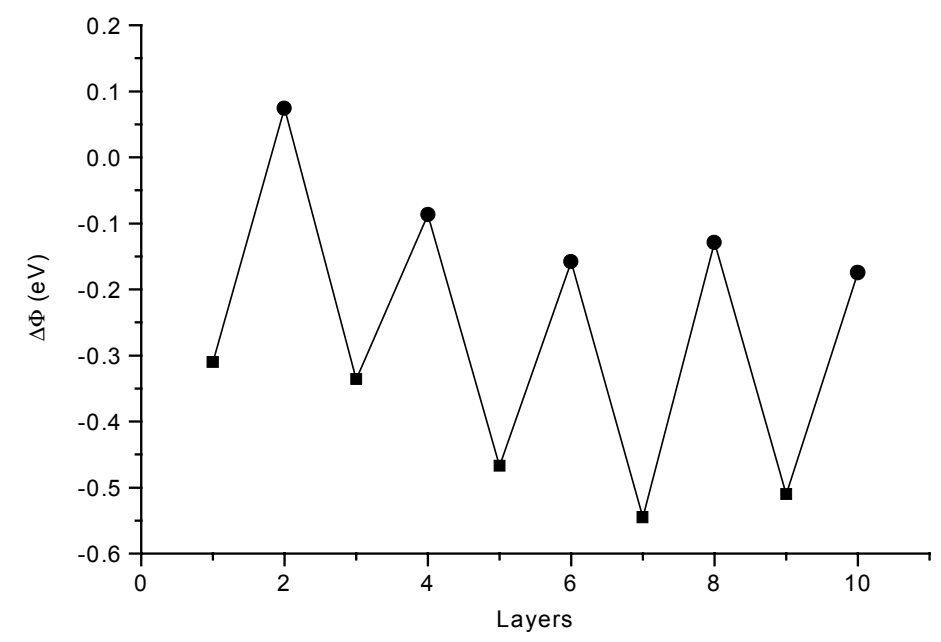

Figure 4. Surface work function $(\phi \mathrm{eV})$ dependence on the terminating layer of OHBP (square) or NiPc (circle) versus the number of total individual layers (i.e., the number of OHBP layers plus NiPc layers).

\subsection{Kevin-Probe Measurements on Multilayer Thin Films}

Contact potential differences (CPDs) were measured after each deposition of viologen oligomers or NiPc layer on ITO. The surface work function values $v s \mathrm{~N}$, indicating that the surface potential of ITO electrodes changed after the deposition of OHBP or NiPc is shown in Figure 4. After a single layer of viologen oligomers was deposited onto an ITO substrate, its CPD value increased by $\sim 300 \mathrm{mV}$ (work function decreased by $0.3 \mathrm{eV}$ ) in reference to blank ITO. After deposition of NiPc onto OHBPcoated ITO, the CPD value decreased to around $50 \mathrm{mV}$ (work function increased by 0.05 $\mathrm{eV}$ ) in reference to blank ITO. Subsequent deposition of OHBP and NiPc layers continue the trend on increasing and decreasing the CPD by $\sim 400 \mathrm{mV}$. The oscillation of the work function with layer deposition demonstrates that the relationship between surface potential and layer deposition (whether OHBP or NiPc) is periodic; therefore, that the surface work function of a multilayer is the same in an ideal situation regardless of the number of bilayers in the structure. We attribute this result to the reproducibility and consistency of the bilayer structure previously observed with UV-vis and FTIR.

Work function change can be due to modification in electron affinity and/or an alteration in band bending at surfaces/interfaces (Bruening et al., 1994; Kronik and Shapira, 1999). Therefore the total change in surface potential is equal to the sum of the modulation in band bending and electron affinity. Since the interaction between OHBP and ITO oxide are weak charge-charge bindings, we expect that changes in the work function from deposition of oppositely charged oligomers or macrocycles on ITO result predominantly from electron affinity contribution. We expect band bending if the selfassembled monolayers have ligand properties that alter the density of the surface states 
(Bruening et al., 1995). The presence of a dipole layer, interacting weakly with surfaces, affects the electron affinity of the substrate only. Both band bending and electron affinity can be changed simultaneously if a dipole layer interacts strongly with surfaces (Bruening et al., 1997; Cohen et al., 1997; Cohen et al., 1999).

Due to the formation of a typical $n$-type depletion layer on bare ITO, there are some negative charges on the surface of ITO, thus promoting the growth of a positively charged viologen monolayer through electrostatic attractions (Moons et al., 1997; Li et al., 2000). The formation of OHBP on ITO surfaces generates a dipole at the inorganicorganic interface, pointing towards ITO, thus lowering the electronic affinity and decreasing its work function. In other words, it is easier for the surface-localized electrons to transfer into the vacuum from the Fermi level after the formation of OHBP/ITO dipole layers. When the negatively charged NiPc layer is deposited on top of a previous OHBP layer, another new dipole layer at the interface of OHBP and NiPc, pointing away from ITO, forms. The net interfacial dipoles of OHBP/ITO and $\mathrm{NiPc} / \mathrm{OHBP}$ cancel each other mostly, and the net CPD values shift back to $50 \mathrm{mV}$, close to the blank reference ITO CPD value. This same effect keeps repeating itself as deposition of OHBP-NiPc multilayers continues. If this modification of the work function were mainly due to an alteration of ITO band bending, we would not have observed the oscillation pattern of CPD values. Therefore, we concluded that this oscillating pattern in the work function was mainly due to modulation in ITO's electron affinity. These results reveal that a Kelvin probe is a very effective tool for monitoring the surface physical properties as multilayer growth is evolving.

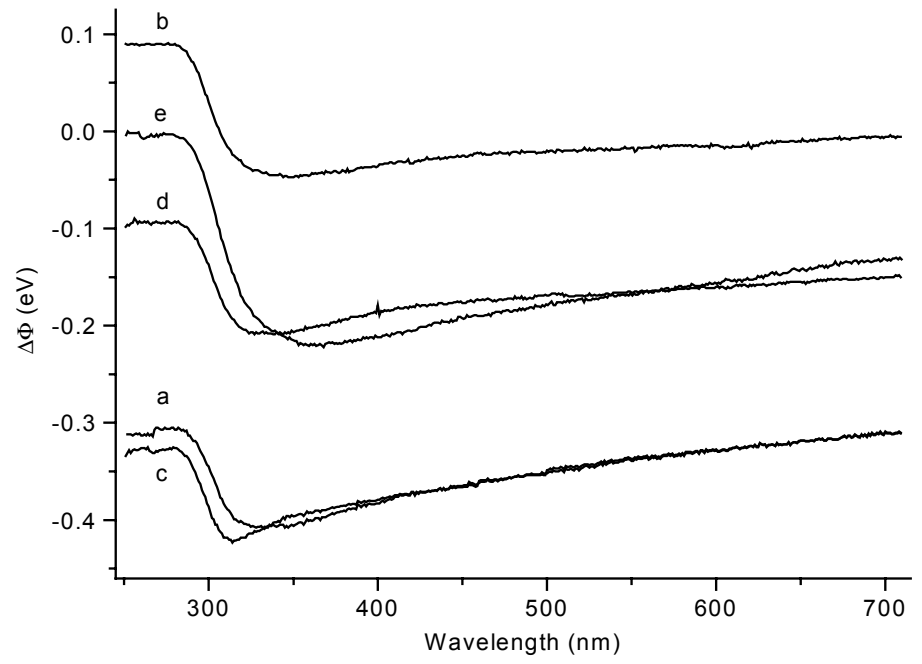

Figure 5. Surface photovoltage spectra (SPS) dependence on the terminating layer of OHBP and NiPc. (a) OHBP/ITO; (b) NiPc-OHBP/ITO; (c) OHBP-NiPc-OHBP/ITO; (d) NiPc-OHBP-NiPc-OHBP/ITO; (e) blank ITO. 


\subsection{Surface Photovoltaic Effects due to Formation of OHBP-NiPc Multilayers}

Surface photovoltage measurement (SPV) is a very sensitive tool to measure the charge redistribution on semiconductor surfaces, buried interfaces, and heterojunctions. Here, we use surface photovoltage spectroscopy (SPS) to study the photovoltaic behaviors of OHBP-NiPc multilayers on ITO (Figure 5). The photo-excited electron-hole pairs will migrate under the intrinsic electric field at the interface, thus changing band bending. Therefore, SPS has been chosen to monitor the band bending change of ITO at the inorganic-organic interface under UV-vis illumination with or without OHBP and NiPc layer modification in this study.

For reference purposes, blank ITO's SPS was employed as the baseline (curve e in Figure 5). It has more than $200 \mathrm{mV}$ photovoltaic response when excited at the band gap $(350 \mathrm{~nm})$ of ITO $\left(\mathrm{E}_{\mathrm{g}}=3.7-4.3 \mathrm{eV}\right)$ (Gerfin and Grätzel, 1996; Hamberg and Granqvist, 1986; Moons et al., 1997), and about $140 \mathrm{mV}$ response when excited at $650 \mathrm{~nm}$. Its negative photovoltaic response value indicates a typical $n$-type material with an upward band bending near the surface. After one layer of electron-accepting oligomeric viologens is assembled, the photovoltaic response is significantly decreased to $105 \mathrm{mV}$ at $350 \mathrm{~nm}$. The reduction of the photovoltaic effect is almost the same at $650 \mathrm{~nm}$ (curves a and e are parallel to each other). This result indicates that surface states have been altered after the deposition of electron accepting OHBP molecules under light illumination. When we grow a NiPc layer and form the first OHBP-NiPc bilayer on ITO, about $30 \mathrm{mV}$ photovoltaic response at $350 \mathrm{~nm}$ was recovered. However, the recovery of photovoltaic effects is $80 \mathrm{mV}$ at $650 \mathrm{~nm}$. The same effect keeps repeating as the deposition of the second OHBP-NiPc bilayers, with the exception that we are losing photovoltaic response with each addition of electron acceptor viologens. The significance of this result is that photo-induced band bending can be really changed by chromophoric monolayer modification on surfaces.

Actually, the total change in surface work function $(\Delta \phi)$ for a semiconductor comes from two terms, the change in electron affinity related to surface dipole, $\Delta \chi$, and the change in band bending, $\Delta \mathrm{V}$ (Bruening et al., 1994):

$$
\Delta \phi=\Delta \chi \pm \Delta \mathrm{V} \text { ( "+" for n-type; “-“ for p-type ) }
$$

Because ITO forms an $n$-type depletion layer, we have $\Delta \phi$ that equals the sum of $\Delta \chi$ and $\Delta \mathrm{V}$. Without light illumination (labeled with superscript (1)), we measured the surface work function change [Eq. (2)], which is shown in Figure 5.

$$
\Delta \phi^{(1)}=\Delta \chi^{(1)}+\Delta \mathrm{V}^{(1)}
$$

With light illumination (labeled with superscript (2)), we measured a photo-induced effect on the change of surface work function [Eq. (3)], which is plotted in Figure 6.

$$
\Delta \phi^{(2)}=\Delta \chi^{(2)}+\Delta \mathrm{V}^{(2)}
$$

Since we expect the electron affinity will remain constant with or without light illumination, i.e., $\Delta \chi^{(1)}=\Delta \chi^{(2)}$, we can deduce the photo-induced band bending change $\Delta \Delta \mathrm{V}=\Delta \mathrm{V}^{(2)}-\Delta \mathrm{V}^{(1)}=\Delta \phi^{(2)}-\Delta \phi^{(1)}$. Accordingly, we have calculated the photo-induced 
band bending change $\Delta \Delta \mathrm{V}$ at the ITO band gap $(350 \mathrm{~nm})$ and in the red region of the spectrum $(650 \mathrm{~nm})$. For bare ITO substrates, we have $\Delta \Delta \mathrm{V}(350 \mathrm{~nm})=-215 \mathrm{meV}$ and $\Delta \Delta \mathrm{V}(650 \mathrm{~nm})=-143 \mathrm{meV}$, which confirms that excitation at the band gap has a more pronounced photovoltaic effect. After the formation of a viologen monolayer, the $\Delta \Delta \mathrm{V}(350 \mathrm{~nm})$ and $\Delta \Delta \mathrm{V}(650 \mathrm{~nm})$ values shift to $-105 \mathrm{meV}$ and $-20 \mathrm{meV}$. When a bilayer of OHBP-NiPc is formed, the photo-induced band bending values are $\Delta \Delta \mathrm{V}(350 \mathrm{~nm})=-$ $137 \mathrm{meV}$ and $\Delta \Delta \mathrm{V}(650 \mathrm{~nm})=-100 \mathrm{meV}$. The second bilayer repeats the same trend. Figure 6 shows the plot of $\Delta \Delta \mathrm{V}(\lambda)$ against excitation wavelength $\lambda$ after the correction from blank ITO, and it is clear that the photo-induced band bending change depends on the terminal layer of the self-assembled multilayers of OHBP-NiPc. In other words, the photo-induced band bending change is also a periodic function of the self-assembled layers, oscillating between two approximate values corresponding to the characteristics of viologen and NiPc.

\section{CONCLUSIONS}

Our results from optical absorption and surface external reflection FTIR reveal that we can grow self-assembled multilayers consisting of electron acceptors like OHBP and donors like NiPc. In order to investigate the evolution of the surface physical properties of self-assembled multilayers, we applied Kelvin probe techniques and surface photovoltage spectroscopy to monitor the growth of self-assembled OHBP and NiPc systems. Kelvin probe results indicate that the surface electronic potential is very sensitive to the presence of self-assembled molecular layers on surfaces. We find that there is an approximately constant gap of $350 \mathrm{meV}$ between the surface work function of OHBP and NiPc terminated self-assembled multilayers. In other words, the surface work function or surface electronic potentials is a periodic function of the terminating layer, oscillating between $\sim 450 \mathrm{mV}$ for OHBP layers and $\sim 100 \mathrm{mV}$ for NiPc layers. On the other hand, the photo-induced effects can be also correlated to the terminating layer of the OHBP-NiPc system. This shows that the self-assembled layers also control the photoinduced effects to some extent. Over the whole excitation spectrum $(300-710 \mathrm{~nm})$, the photo-induced band bending change oscillates between two values mostly governed by the properties of the terminating layer of the self-assembled systems.

\section{ACKENOWLEDGMENTS.}

LSL acknowledge the support of the Laboratory Directed Research and Development programs. ADQL acknowledges the support of Center for Materials Science, Department of Chemistry, and College of Science at Washington State University. 


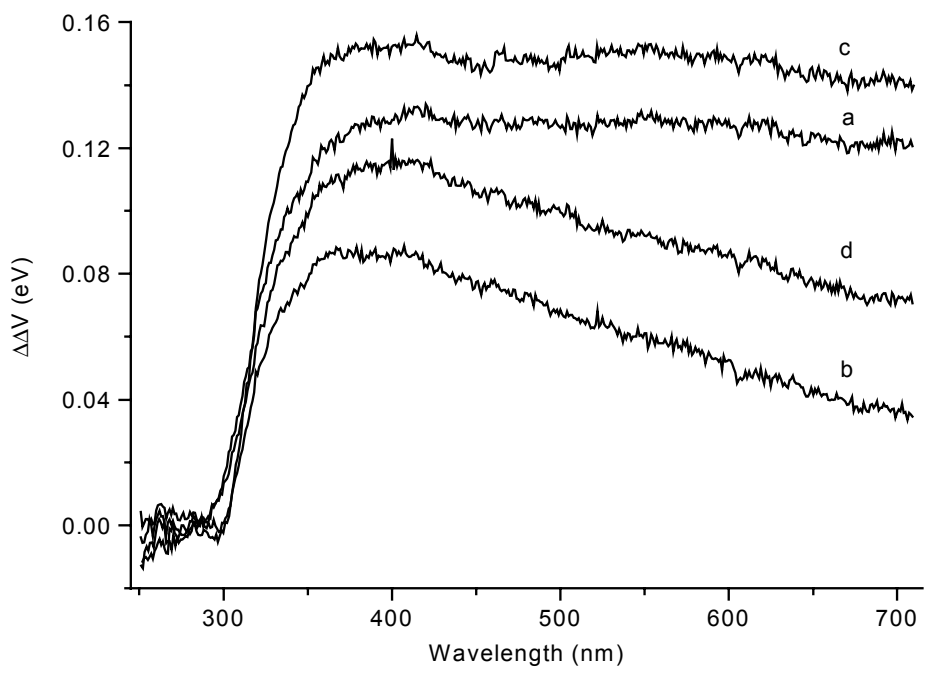

Figure 6. $\Delta \Delta \mathrm{V}(\mathrm{mV})$ dependence on the excitation wavelength $\lambda$ after the correction from ITO, i.e. $\phi_{\mathrm{sam}}-\phi_{\mathrm{ITO}}$ : (a) OHBP/ITO; (b) NiPc-OHBP/ITO; (c) OHBP-NiPc-OHBP/ITO; (d) NiPc-OHBP-NiPc-OHBP/ITO.

\section{REFERENCES:}

Bae, I. T., Huang, H., Yeager, E. B., and Scherson, D. A., 1991, In-situ infrared spectroscopic studies of redox active self-assembled monolayers on gold electrode surfaces, Langmuir 7: 1558.

Baikie, I. D., and Estrup, P. J. 1998, Low cost PC based scanning Kelvin probe, Rev. Sci. Instrum. 69:3902.

Bastide, S., Butruille, R., Cahen, D., Dutta, A., Libman, J., Shanzer, A., Sun L., and Vilan, A., 1997, Controlling the work function of GaAs by chemisorption of benzoic acid derivatives, J. Phys. Chem. B 101: 2678 .

Bruening, M., Cohen, R., Guillemoles, J. F., Moav, T., Libman, J., Shanzer, A., and Cahen, D, 1997, Simultaneous control of surface potential and wetting of solids with chemisorbed multifunctional ligands, J. Am. Chem. Soc. 119:5720.

Bruening, M., Moons, E., Cahen, D., and Shanzer, A., 1995, Controlling the work function of CdSe by chemisorption of benzoic-acid derivatives and chemical etching, J. Phys. Chem. 99: 8368.

Bruening, M., Moons, E., Yaron-Marcovich, D., Cahen, D., Libman, J., and Shanzer, A., 1994, Polar ligand adsorption controls semiconductor surface-potentials, J. Am. Chem. Soc. 116: 2972.

Christensen, P. A., and Hamentt, A., 1989, An in-situ FTIR study into the nature of completely reduced $\mathrm{MV}^{2+}$, J. Electroanal. Chem. 263:49.

Cohen, R., Bastide, S., Cahen, D., Libman, J., Shanzer, A., and Rosenwaks, Y., 1997, Controlling electronic properties of CdTe by adsorption of dicarboxylic acid derivatives: Relating molecular parameters to band bending and electron affinity changes, Adv. Mater. 9: 746.

Cohen, R., Kronik, L., Shanzer, A., Cahen, D., Liu, A., Rosenwakes, Y., Lorenz, J. K., and Ellis, A. B., 1999, Molecular control over semiconductor surface electronic properties: Dicarboxylic acids on CdTe, CdSe, GaAs, and InP, J. Am. Chem. Soc. 121: 10545.

Cohen, R., Kronik, L., Vilan, A., Shanzer, A., and Cahen, D., 2000, Frontier orbital model of semiconductor surface passivation: Dicarboxylic acids on n- and p-GaAs, Adv. Mater. 12: 33.

Colthup, N. B., Daly L. H., and Wiberley, S. E., 1975, Introduction to Infrared and Raman Spectroscopy, $2^{\text {nd }}$ ed., Academic Press, New York, p. 355. 
Dante, S., Hou, Z., Risbud S., and Stroeve, P., 1999, Nucleation of iron oxy-hydroxide nanoparticles by layerby-layer polyionic assemblies, Langmuir 15: 2176.

Decher, G., 1997, Fuzzy nanoassemblies: Toward layered polymeric multicomposites, Science 277: 1232.

Enokida, T., and Hirohashi, R., 1991, A new synthesis of epsilon-from nickel phthalocyanine by using DBU at low-temperature, Chem. Lett. 12:2155.

Fukui, M., Katayama, N., Ozaki, Y., Araki, T., and Iriyama, K., 1991, Structural characterization of phthalocyanine Langmuir-Bloadgett multiplayer assemblies by FT-IR spectroscopy, Chem. Phys. Lett. 177: 247.

Gerfin, T., and Grätzel, M., 1996, Optical properties of tin-doped indium oxide determined by spectroscopic ellipsometry, J. Appl. Phys. 79:1722.

Gong, Y. K., Miyamoto, T., Nakashima, K., and Hashimoto, S., 2000, Photoinduced electron transfer from 3(9-anthracene)propyltrimethyl ammonium bromide and pyrene to methyl viologen on the surface of polystyrene latex particles, J. Phys. Chem. B 104:5772.

Hamberg, I., and Granqvist, C. G., 1986, Vaporated Sn-doped $\operatorname{In}_{2} \mathrm{O}_{3}$ films: Basic optical-properties and applications to energy-efficient widows, J. Appl. Phys. 60:R123.

Hamma, S., and Cabarrocas, P. R., 1999, Determination of the mobility gap of microcrystalline silicon and of the band discontinuities at the amorphous microcrystalline silicon interface using in situ Kelvin probe technique, Appl. Phys. Lett. 74: 3218.

Hanack, M., and Lang, M., 1994, Conducting stacked metallophthalocyanines and related-compounds, Adv. Mater. 6: 819

He, J. A., Bian, S., Li, L., Kumar, J., Tripathy, S. K., and Samuelson, L. A. . J. Phys. Chem. B 2000, 104, 10513.

Kaschak, D. M., Lean, J. T., Waraksa, C. C., Geoffrey, B. S., Usami, H., and Mallouk, T. E., 1999, Photoinduced energy and electron transfer reactions in lamellar polyanion/polycation thin films: Toward an inorganic "leaf", J. Am. Chem. Soc. 121: 3435.

Kelly, L. A., and Rodgers, M. A. J., 1994, Reductive quenching of novel mixed-ligand tris(bipyridyl) ruthenium(II) complexes in aqueous-solution and inert colloidal suspensions, J. Phys. Chem. 98:6377.

Kim, J. Wang, H. C., Kumar, J., Tripathy, S. K., Chittibabu, K. G., Cazeca M. J., and Kim W., 1999, Novel layer-by-layer complexation technique and properties of the fabricated films, Chem. Mater. 11: 2250.

Kronik, L., and Shapira, Y., 1999, Surface photovoltage phenomena: theory, experiment, and applications, Surface Science Reports 37: 1.

Le, T. P., Rogers, J. E., and Kelly, L. A., 2000, Photoinduced electron transfer in covalently linked 1,8naphthalimide/viologen systems, J. Phys. Chem. A 104: 6778.

Lenahan, K. M., Wang, Y. X., Liu, Y. J., Claus, R. O., Heflin, J. R., Marciu, D., and Figura C., 1998, Novel polymer dyes for nonlinear optical applications using ionic self-assembled monolayer technology, $A d v$. Mater. 10: 853.

Li, D. Q., Bishop, A., Gim, Y., Shi, X. B., Fitzsimmons, M. R., and Jia, Q. X., 1998, Conduction properties of metal organic monolayer semiconductor heterostructures, Appl. Phys. Lett. 73: 2645.

Li, D. Q., Lütt, M., Fitzsimmons, M. R., Synowicki, R., Hawley, M. E., and Brown G. W., 1998, Preparation, characterization, and properties of mixed organic and polymeric self-assembled multilayers, J. Am. Chem. Soc. 120: 8797.

Li, L. S., Wang, R., Fitzsimmons, M., and Li, D. Q., 2000, Surface electronic properties of self-assembled, oppositely charged macrocycle and polymer multilayers on conductive oxides, J. Phys. Chem. B 104: 11195.

Liu, Y. J., and Claus, R. O., 1999, Strong enhancement of optical absorbency from ionic self-assembled multiplayer thin-films of nanocluster Pt and polymer dye, J. Appl. Phys. 85: 419.

Liu, Y. J., Wang A., and Claus, R. O., 1997, Molecular self-assembly of $\mathrm{TiO}_{2}$ /polymer nanocomposite films, $J$. Phys. Chem. B 101: 1385.

Lütt, H., 1993, Surfaces and interfaces of solids, Springer-Verlag, Berlin Heidelberg, pp. 464-471.

Lütt, M., Fitzsimmons, M. R., and Li, D. Q., 1998, X-ray reflectivity study of self-assembled thin films of macrocycles and macromolecules, J. Phys. Chem. B 102: 400.

Moons, E., Goossens, A., and Savenije, T., 1997, Surface photovoltage of porphyrin layers using the Kelvin probe technique, J. Phys. Chem. B 101: 8492.

Ostrander, J. W., Mamedov, A. A., and Kotov N. A., 2001, Two modes of linear layer-by-layer growth of nanoparticle-polylectrolyte multilayers and different interactions in the layer-by-layer deposition, J. Am. Chem. Soc. 123: 1101.

Schlenoff, J. B., Laurent, D., Ly, H., and Stepp, J., 1998, Redox-active polyelectrolyte multilayers, Adv. Mater. 10: 347 . 
Smolenyak, P., Peterson, R., Nebesny, K., Torker, M., O’Brien, D. F., and Armstrong, N. R., 1999, Highly ordered thin films of octasubstituted phthalocyanines, J. Am. Chem. Soc. 121: 8628 .

Stymne, B., Sauvage, F. X., and Wettermark, G., 1979, Spectroscopic study of the complexation of phthalocyanines with water, ethanol and phenol, Spectrochim. Acta 35A: 1195.

Tadayyon, S. M., Griffiths, K., Norton, P. R., Tripp, C., and Popovic, Z., 1999, Work function modification of indium-tin-oxide used in organic light emitting devices, J. Vac. Sci. Technol. A 17: 1773.

Watanabe, T., Ama, T., and Nakamoto, K., 1983, Matrix-isolation infrared-spectra of (octaethylporphyrinato) manganese(II) and (phthalocyaninato)manganese(II) and their dioxygen adducts, Inorg. Chem. 22: 2470. 